\title{
Adaptive integration of constitutive rate equations
}

\author{
Wolfgang Fellin* and Markus Mittendorfer ${ }^{\dagger}$ and Alexander Ostermann ${ }^{\ddagger}$
}

27 November 2008

post-print of:

Fellin, Wolfgang; Mittendorfer, Markus; Ostermann, Alexander

(2009): Adaptive integration of constitutive rate equations. In: Computers and Geotechnics 36/5, pp. 698 - 708.

\begin{abstract}
In finite element calculations the constitutive model plays a key role. The evaluation of the stress response of the constitutive relation for a given strain increment, which is a time integration in the case of models of the rate type, is a typical sub task in such calculations. Adaptive behaviour of the time integration is essential to assure numerical stability and to control the accuracy of the solution. An adaptive second order semi-implicit method is developed in this paper. Its numerical behaviour is compared with an adaptive second order explicit scheme. The two proposed methods control the local error and guarantee numerical stability of the time integration. We include several numerical geotechnical element tests using hypoplasticity with intergranular strain. The element tests simulate the behaviour of a finite element method based on the displacement formulation.
\end{abstract}

Kepywords: Hypoplasticity; intergranular strain; consistent tangent operators; error control; finite elements

\section{Introduction}

The computation of the stress response of a constitutive relation for a given strain increment is a typical sub task in finite element calculations. Constitutive models of the rate type require a time integration procedure for this task. In order to save computational time, the given strain increments used in finite

\footnotetext{
*Unit of Geotechnical and Tunnel Engineering, Department of Infrastructure, University of Innsbruck

${ }^{\dagger}$ Department of Mathematics, University of Innsbruck

${ }_{\ddagger}^{\ddagger}$ Department of Mathematics, University of Innsbruck
} 
element programs are usually rather large. Therefore, the time integration has to employ some sub stepping in order to account for the nonlinearity of the constitutive model.

Early versions of finite element implementations were based on the explicit Euler method with constant step sizes, e.g., [10, 21]. Later implementations $[7,19,2]$ used the implicit Euler method. Semi-implicit Rosenbrock methods were employed in [1]. In all these methods, the integration error is not controlled. In our opinion, however, a reliable error control is essential for the integration of nonlinear constitutive relations as it is the basis for an adaptive step size selection. Higher order explicit methods with step size control were applied in $[4,22]$.

In this paper we develop an adaptive second order semi-implicit method and compare its numerical behaviour with that of the explicit method of [4]. Hypoplasticity with intergranular strain [20] is chosen for this comparison. Under certain circumstances, this constitutive model can exhibit a numerically stiff behaviour for which implicit methods are known to be superior. We use a semi-implicit extrapolation scheme. In contrast to fully implicit methods, semiimplicit methods are much faster in computation, although they require slightly more sub steps.

The present article is organized as follows. In Section 2 we shortly introduce the notation of constitutive models of the rate type. In Section 3 we present two time integration methods and describe an error control on which the adaptive sub step size strategy is based. Section 4 illustrates our two approaches with various numerical examples. We have chosen four typical geotechnical element tests: the confined compression test, the drained and the undrained triaxial test, as well as the constant volume simple shear test. Our main conclusions are finally given in Section 5. To make our paper self-contained, we outline the used constitutive model in Appendix A and give some implementation details in Appendix B.

\section{Constitutive models of the rate type}

A core part of any mechanical model of a continuum, mathematically formulated as an initial boundary value problem, is the description of the mechanical behaviour of the material. The latter can be provided by a constitutive model of the rate type. Such models are relations of the objective stress rate $\stackrel{\mathrm{T}}{\mathbf{T}}$ of the effective Cauchy stress [23] with the Eulerian stretching D, the effective Cauchy stress $\mathbf{T}$ and some additional state or internal variables $\mathbf{Q}$

$$
\stackrel{\circ}{\mathbf{T}}=\mathbf{h}(\mathbf{T}, \mathbf{D}, \mathbf{Q}) \text {. }
$$

The additional state variables obey a further set of evolution equations

$$
\stackrel{\mathrm{Q}}{=} \mathbf{k}(\mathbf{T}, \mathbf{D}, \mathbf{Q}) \text {. }
$$

Typical representatives of such models for geotechnical applications are the various versions of hypoplasticity, e.g. $[11,24,20,9,14,15,17,25]$, or the 
CloE-model $[3,16]$. We use hypoplasticity with intergranular strain [20] to test the performance of the numerical schemes proposed. This model is outlined in Appendix A.

Note that elasto-plastic constitutive models can be formulated in rate form, too. Mathematically, this leads to a system of differential equations with algebraic constraints (e.g. yield surfaces). For such systems, appropriate numerical integrators exist [6]. Such integration methods were already applied successfully for one-dimensional formulations of elasto-plastic models in [18]. There, problems with a nonlinear elastic core and nonlinear hardening were considered.

\section{Adaptive integration}

Collecting the components of the stress tensor $\mathbf{T}$, the additional state variables $\mathbf{Q}$, and all derivatives with respect to the stretching in a vector $\mathbf{y}$, we obtain a nonlinear system of differential equations

$$
\begin{aligned}
\mathbf{y}^{\prime}(t) & =\mathbf{f}(\mathbf{y}(t)) \\
\mathbf{y}(0) & =\mathbf{y}_{0}
\end{aligned}
$$

with $m$ components. In this section, we explain in detail how we solve this initial value problem numerically.

In many scientific applications, the explicit Euler method

$$
\mathbf{y}_{n+1}=\mathbf{y}_{n}+\tau_{n} \mathbf{f}\left(\mathbf{y}_{n}\right)
$$

is still the method of choice for integrating (3). Notwithstanding its computational simplicity, the explicit Euler method has several shortcomings:

- It is first order accurate only and therefore might require a large number of steps to meet the accuracy requirements.

- It is not suited for stiff problems.

- It does not supply an estimate of the committed errors. Such an error estimate, however, is indispensable for selecting the time step size $\tau_{n}$ adaptively.

\subsection{A second order explicit method}

We first recall how a second order method can be constructed from single Euler steps by extrapolation. Let $\widetilde{\mathbf{y}}_{n}$ be the numerical approximation to the exact solution of (3) at time $t_{n}$, and let $\mathbf{z}(t)$ denote the exact solution of (3a) passing through this value, i.e.

$$
\mathbf{z}\left(t_{n}\right)=\widetilde{\mathbf{y}}_{n}
$$

see Figure 1. To obtain a numerical approximation at time $t_{n+1}=t_{n}+\tau_{n}$, we perform one Euler step of size $\tau_{n}$

$$
\mathbf{v}=\widetilde{\mathbf{y}}_{n}+\tau_{n} \mathbf{f}\left(\widetilde{\mathbf{y}}_{n}\right)
$$




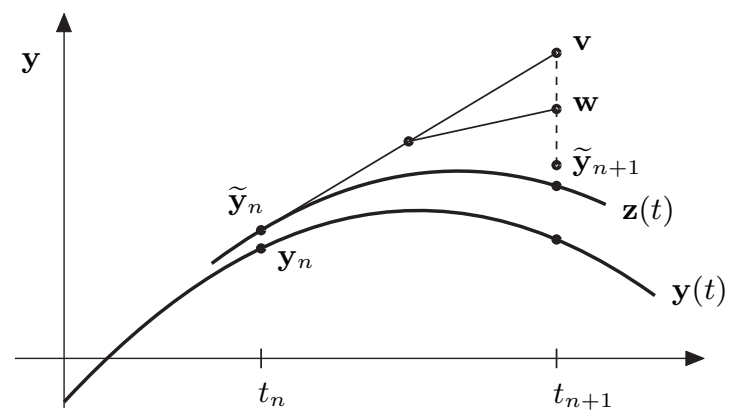

Figure 1: Euler steps and extrapolated value $\widetilde{\mathbf{y}}_{n+1}$.

as well as two Euler steps of size $\tau_{n} / 2$

$$
\mathbf{w}=\widetilde{\mathbf{y}}_{n}+\frac{\tau_{n}}{2} \mathbf{f}\left(\widetilde{\mathbf{y}}_{n}\right)+\frac{\tau_{n}}{2} \mathbf{f}\left(\widetilde{\mathbf{y}}_{n}+\frac{\tau_{n}}{2} \mathbf{f}\left(\widetilde{\mathbf{y}}_{n}\right)\right) .
$$

A straightforward Taylor expansion shows that both values are first order approximations

$$
\begin{aligned}
\mathbf{v} & =\mathbf{z}\left(t_{n+1}\right)-\frac{1}{2} \mathbf{f}^{\prime}\left(\widetilde{\mathbf{y}}_{n}\right) \mathbf{f}\left(\widetilde{\mathbf{y}}_{n}\right) \tau_{n}^{2}+\mathcal{O}\left(\tau_{n}^{3}\right), \\
\mathbf{w} & =\mathbf{z}\left(t_{n+1}\right)-\frac{1}{4} \mathbf{f}^{\prime}\left(\widetilde{\mathbf{y}}_{n}\right) \mathbf{f}\left(\widetilde{\mathbf{y}}_{n}\right) \tau_{n}^{2}+\mathcal{O}\left(\tau_{n}^{3}\right) .
\end{aligned}
$$

Their combination

$$
\widetilde{\mathbf{y}}_{n+1}=2 \mathbf{w}-\mathbf{v}=\mathbf{z}\left(t_{n+1}\right)+\mathcal{O}\left(\tau_{n}^{3}\right),
$$

however, is a second order approximation to the local solution $\mathbf{z}(t)$. The discretisation error after one step

$$
\tilde{\mathbf{y}}_{n+1}-\mathbf{z}\left(t_{n+1}\right)=\mathcal{O}\left(\tau_{n}^{3}\right),
$$

is called local error of the method. Consequently, a standard argument [5] shows that the global error of the method is bounded by

$$
\left\|\widetilde{\mathbf{y}}_{n}-\mathbf{y}\left(t_{n}\right)\right\| \leq C \tau^{2}
$$

where $\tau=\max \tau_{n}$. The constant $C$ is independent of $n$ and the chosen step size sequence, but it depends on the differential equation and on the length of the time interval $0 \leq t_{n} \leq t_{\text {end }}$.

The resulting method (7) is called Richardson extrapolation of the explicit Euler method. This method coincides with the classical two stage Runge-Kutta method, given by Runge in 1895. In the remainder of this paper, we will call this method ERK2. 


\subsection{Error estimation and step size control}

Next, we will treat the problem of step size selection. Our approach is that of $[4,5]$. Let

$$
C=\frac{1}{2}\left\|\mathbf{f}^{\prime}\left(\widetilde{\mathbf{y}}_{n}\right) \mathbf{f}\left(\widetilde{\mathbf{y}}_{n}\right)\right\|
$$

denote the error constant of the Euler method. The difference of the auxiliary values $(6)$

$$
\mathrm{EST}=\|\mathbf{w}-\mathbf{v}\|=\frac{C}{2} \tau_{n}^{2}+\mathcal{O}\left(\tau_{n}^{3}\right)
$$

is an asymptotically correct estimate for the local error of w. For a user-supplied tolerance TOL, the optimal step size $\tau_{\text {opt }}$ in the present step would have been

$$
\frac{C}{2} \tau_{\mathrm{opt}}^{2}=\mathrm{TOL}
$$

Dividing (10) by (9) yields

$$
\tau_{\mathrm{opt}}=\tau_{n} \sqrt{\frac{\mathrm{TOL}}{\mathrm{EST}}}
$$

We use this for controlling the step size. If the estimated error EST is below the tolerance TOL, the step is accepted and the new step size

$$
\tau_{\text {new }}=\tau_{n} \cdot \min \left(\kappa_{\mathrm{I}}, \max \left(\kappa_{\mathrm{D}}, 0.9 \cdot \sqrt{\frac{\mathrm{TOL}}{\mathrm{EST}}}\right)\right)
$$

is chosen for the next step. If the estimated error EST is larger than TOL, however, we reject the step and redo it with a smaller step size, given again by $(11)$.

The factor 0.9 in (11) is a safety factor that accounts for the neglected higher order terms of (9). The other two constants $\kappa_{\mathrm{D}}$ and $\kappa_{\mathrm{I}}$ limit the step size change (maximum decrease and increase, respectively). A typical choice is $\kappa_{\mathrm{D}}=0.2$ and $\kappa_{\mathrm{I}}=2$.

In order to obtain a reliable error estimate, it is common to use a scaled Euclidian norm in (9)

$$
\|\mathbf{v}-\mathbf{w}\|^{2}=\frac{1}{m} \sum_{i=1}^{m}\left(\frac{v_{i}-w_{i}}{s_{i}}\right)^{2}
$$

with the scaling factors

$$
s_{i}=a_{i}+r_{i} \cdot \max \left(\left|\left(\widetilde{\mathbf{y}}_{n}\right)_{i}\right|,\left|\left(\widetilde{\mathbf{y}}_{n+1}\right)_{i}\right|\right) .
$$

The parameters $a_{i}$ and $r_{i}$ are used to fine-tune the error estimate. Taking $a_{i}=0$ and $r_{i}=1$ results in a relative error estimate. This is important when the absolute value of the corresponding quantity (a stress component, e.g.) gets considerably larger than 1 . On the other hand, this choice is dangerous whenever 
the solution gets close to zero. In the latter case, the absolute error should be controlled.

Let $\mathrm{AERR}_{i}$ be the largest absolute error of component $i$ accepted by the user. Then the choice

$$
a_{i}=\frac{\mathrm{AERR}_{i}}{\mathrm{TOL}}
$$

enforces that steps with errors larger than $\mathrm{AERR}_{i}$ (in component $i$ ) will be rejected. A one-dimensional example illustrating the effect of AERR on the step size selection is given in Table 1 .

In the experiments below, we prescribe TOL and $\mathrm{AERR}_{i}$ according to our requirements, and we choose $r_{i}=1$.

\begin{tabular}{|r|r|c|c|}
\hline \multicolumn{1}{|c|}{$\mathrm{V}$} & \multicolumn{1}{|c|}{$\mathrm{w}$} & AERR=10 & AERR=1 \\
\hline 100,005 & 100,000 & accepted & accepted \\
10,005 & 10,000 & accepted & accepted \\
1,005 & 1,000 & accepted & rejected \\
105 & 100 & accepted & rejected \\
\hline
\end{tabular}

Table 1: One-dimensional example $(m=1)$ of error control with TOL $=10^{-3}$ and $r=1$ : the step is accepted or rejected depending on the size of AERR.

\subsection{A second order semi-implicit method}

Explicit integrators get inefficient when (3) becomes stiff. A remedy would be to replace the basic integrator (4) by the fully implicit Euler method

$$
\mathbf{y}_{n+1}=\mathbf{y}_{n}+\tau_{n} \mathbf{f}\left(\mathbf{y}_{n+1}\right) .
$$

This method has very good stability properties. Its implementation, however, requires the solution of systems of nonlinear equations. The latter can be a time consuming task.

As a compromise, we propose to consider the semi-implicit Euler method

$$
\mathbf{y}_{n+1}=\mathbf{y}_{n}+\tau_{n} \mathbf{f}\left(\mathbf{y}_{n}\right)+\tau_{n} \mathbf{f}^{\prime}\left(\mathbf{y}_{n}\right)\left(\mathbf{y}_{n+1}-\mathbf{y}_{n}\right)
$$

whose format is motivated by truncated Taylor series expansion of the right hand side of (14). The semi-implicit Euler method can be written equivalently as

$$
\left[\mathbf{I}-\tau_{n} \mathbf{f}^{\prime}\left(\mathbf{y}_{n}\right)\right]\left(\mathbf{y}_{n+1}-\mathbf{y}_{n}\right)=\tau_{n} \mathbf{f}\left(\mathbf{y}_{n}\right) .
$$

In contrast to the implicit Euler method, it only requires the solution of linear systems of equations. Starting from this basic integration step, we construct the auxiliary values $\mathbf{v}$ and $\mathbf{w}$ as before. From

$$
\left[\mathbf{I}-\tau_{n} \mathbf{f}^{\prime}\left(\widetilde{\mathbf{y}}_{n}\right)\right]\left(\mathbf{v}-\widetilde{\mathbf{y}}_{n}\right)=\tau_{n} \mathbf{f}\left(\widetilde{\mathbf{y}}_{n}\right)
$$


we obtain by Taylor series expansion

$$
\mathbf{v}=\widetilde{\mathbf{y}}_{n}+\tau_{n} \mathbf{f}\left(\widetilde{\mathbf{y}}_{n}\right)+\tau_{n}^{2} \mathbf{f}^{\prime}\left(\widetilde{\mathbf{y}}_{n}\right) \mathbf{f}\left(\widetilde{\mathbf{y}}_{n}\right)+\mathcal{O}\left(\tau_{n}^{3}\right) .
$$

In the same way, the defining equations for $\mathbf{w}$

$$
\begin{aligned}
{\left[\mathbf{I}-\frac{\tau_{n}}{2} \mathbf{f}^{\prime}\left(\widetilde{\mathbf{y}}_{n}\right)\right]\left(\mathbf{w}^{\star}-\widetilde{\mathbf{y}}_{n}\right) } & =\frac{\tau_{n}}{2} \mathbf{f}\left(\widetilde{\mathbf{y}}_{n}\right), \\
{\left[\mathbf{I}-\frac{\tau_{n}}{2} \mathbf{f}^{\prime}\left(\widetilde{\mathbf{y}}_{n}\right)\right]\left(\mathbf{w}-\mathbf{w}^{\star}\right) } & =\frac{\tau_{n}}{2} \mathbf{f}\left(\mathbf{w}^{\star}\right) .
\end{aligned}
$$

imply that

$$
\mathbf{w}=\widetilde{\mathbf{y}}_{n}+\tau_{n} \mathbf{f}\left(\widetilde{\mathbf{y}}_{n}\right)+\tau_{n}^{2} \frac{3}{4} \mathbf{f}^{\prime}\left(\widetilde{\mathbf{y}}_{n}\right) \mathbf{f}\left(\widetilde{\mathbf{y}}_{n}\right)+\mathcal{O}\left(\tau_{n}^{3}\right) .
$$

In order to reduce the numerical work, we have used the same Jacobian $\mathbf{f}^{\prime}\left(\widetilde{\mathbf{y}}_{n}\right)$ in all three steps. The extrapolated value

$$
\widetilde{\mathbf{y}}_{n+1}=2 \mathbf{w}-\mathbf{v}=\mathbf{z}\left(t_{n+1}\right)+\mathcal{O}\left(\tau_{n}^{3}\right),
$$

is again a second order approximation to the local solution $\mathbf{z}(t)$. It is called Richardson extrapolation of the semi-implicit Euler method. In the remainder of this paper, we will call this method SIRK2. The step size selection for this method is performed as described in Section 3.2.

\section{Element tests}

The performance of the proposed time integration schemes is shown for typical element tests from geotechnical engineering. The computations were performed with MATLAB. We have chosen three tests with rectilinear extensions: the confined compression test, the drained and the undrained triaxial test. The fourth test is the constant volume simple shear test in which rotations of the stress tensor occur.

\subsection{Layout of the program}

We have chosen a modular set up for our program, which is based on operator splitting. Such an approach is standard in finite element programs. The element tests were conducted on a stress-strain level in a strain controlled manner, i.e. a load increment is defined in a main program by a rotational free strain increment $\Delta \varepsilon$ which is assumed to be constant over a time window from $t_{a}$ to $t_{a}+\Delta t$. The main program passes the actual Cauchy stress tensor $\mathbf{T}\left(t_{a}\right)$ and the time increment $\Delta t$ to a constitutive subroutine which integrates the constitutive equations with a fully given stretching tensor

$$
\mathbf{D}=\frac{\Delta \varepsilon}{\Delta t}
$$

using the proposed error controlled time sub stepping. The constitutive subroutine returns the co-rotational part of the Cauchy stress tensor at the end of the 
time step $\mathbf{T}\left(t_{a}\right)+\stackrel{\circ}{\mathbf{T}}\left(t_{a}\right) \cdot \Delta t$. Note that the objective stress rate $\stackrel{\circ}{\mathbf{T}}$ is equal to the time rate $\dot{\mathbf{T}}$ for rectilinear tests or for small strains, and, therefore, the stress update of the constitutive subroutine can directly be used. For the simple shear test, this does not hold any more. However, as large strain effects are usually treated by the finite element code, we apply a simple forward integration of the stress. We define the objective stress rate as the Zaremba-Jaumann stress rate

$$
\stackrel{\circ}{\mathbf{T}}=\dot{\mathbf{T}}-\mathbf{W} \mathbf{T}+\mathbf{T W},
$$

where $\mathbf{W}$ is the spin tensor. This was proposed, e.g., in [13]. The stress $\mathbf{T}$ is then updated at the end of each load step with a forward Euler step

$$
\begin{aligned}
\mathbf{T}\left(t_{a}+\Delta t\right)= & \mathbf{T}\left(t_{a}\right)+\stackrel{\circ}{\mathbf{T}}\left(t_{a}\right) \cdot \Delta t \\
& +\Delta t\left(\mathbf{W}\left(t_{a}\right) \mathbf{T}\left(t_{a}\right)-\mathbf{T}\left(t_{a}\right) \mathbf{W}\left(t_{a}\right)\right),
\end{aligned}
$$

wherein the first two terms on the right hand side represent the co-rotational update of the Cauchy stress tensor performed by the constitutive subroutine. An equivalent approach was applied to tensorial additional state variables.

For mixed boundary conditions, the main program performs Newton iterations for the unknown strain components with an initial guess $\Delta \varepsilon^{0}$ for the strain increment. In this case, the constitutive subroutine has to provide the derivative of the stress with respect to the strain increment

$$
\frac{\partial \mathbf{T}\left(t_{a}+\Delta t\right)}{\partial \Delta \varepsilon}=\frac{\partial \Delta \mathbf{T}}{\partial \Delta \varepsilon},
$$

the so-called Jacobian, which is calculated with the method presented in [4], compare Appendix B. Based on this information, a new guess for the strain increment is calculated and the whole procedure is iterated until convergence.

\subsection{Numerical settings}

The following values for the tolerances are used in all numerical experiments: TOL $=10^{-3}$ and

$$
\mathrm{AERR}_{\mathbf{T}}=0.1, \quad \mathrm{AERR}_{e}=0.01, \quad \mathrm{AERR}_{\boldsymbol{\delta}}=10^{-7} .
$$

The error estimate (12) is calculated using the state variables only, i.e. $m=13$, compare Appendix B.1.

An exact solution for each test was obtained with $10^{4}$ load steps with the explicit Euler method to calculate the global errors of the state variables. This solution is plotted as continuous line in the following figures, whereas the numerical solutions are plotted as symbols.

\subsection{Confined compression test}

We first investigate a confined compression test sketched in Figure 2. The initial stresses are $T_{11}=-100 \mathrm{kN} / \mathrm{m}^{2}, T_{22}=T_{33}=-50 \mathrm{kN} / \mathrm{m}^{2}$, the initial void ratio is $e=0.735$ and the initial intergranular strains are zero. 


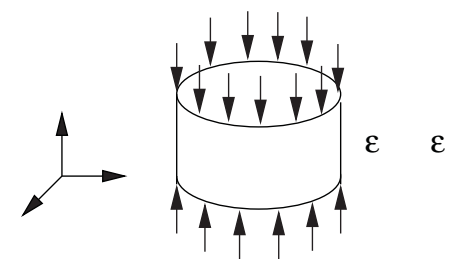

Figure 2: Confined compression test.

A loading was performed in one step with $\Delta \varepsilon_{11}=-1.5 \times 10^{-2}$, then unloading in one step with $\Delta \varepsilon_{11}=1.5 \times 10^{-3}$ and reloading in one step with $\Delta \varepsilon_{11}=$ $-4 \times 10^{-3}$. The resulting stress-strain relation is shown in Figure 3. During the first loading, the intergranular strain reaches its extremum $\delta_{11}=-R$, which cannot be exceeded by further deformation in the same direction, see Figure 4. During unloading $\delta_{11}$ increases again up to $+R$.

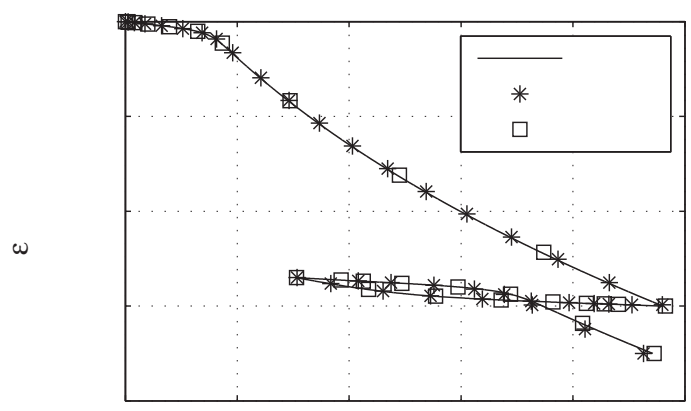

Figure 3: Confined compression test: stress-strain relationship in a loadingunloading-reloading cycle; crosses and squares mark every third sub step and the final solution with ERK2 and SIRK2, respectively.

The adaptivity of both methods becomes obvious by the varying distances between consecutive symbols in Figures 3 and 4 . These symbols mark every third sub step of the time integration. Furthermore, they illustrate that the semi-implicit time integration SIRK2 uses larger sub steps than the explicit method ERK2, especially in the region where $\delta_{11}$ has reached the limit $-R$.

The absolute errors of $T_{11}$ in the first loading are in the same order for both methods (Figure 5), but the semi-implicit integration exhibits a more stable behaviour. In the confined compression test, the error of $T_{11}$ is mainly controlled by the error of $\delta_{11}$, which can be seen by a comparison of the relative errors 


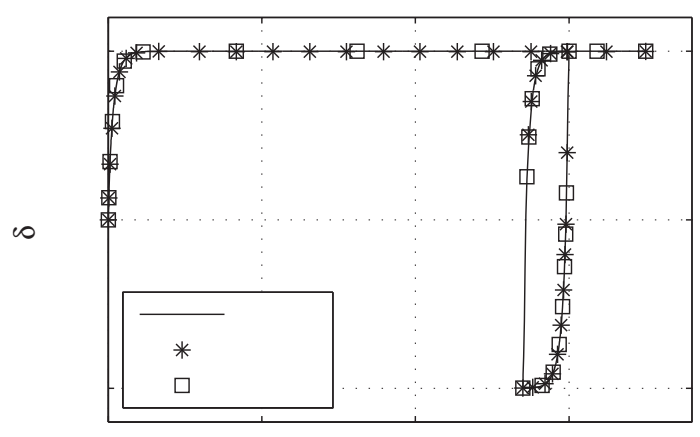

$\varepsilon$

Figure 4: Confined compression test: evolution of the intergranular strain: loading-unloading-reloading cycle; crosses and squares mark every third sub step with ERK2 and SIRK2, respectively.

of $T_{11}$ and $\delta_{11}$ of the explicit method in Figure 6 . The error of $\delta_{11}$ shows an unstable behaviour in the region where $\delta_{11}$ has to stay at the limit value $-R$. This indicates a stiff behaviour of the evolution equation of the intergranular strain (31).

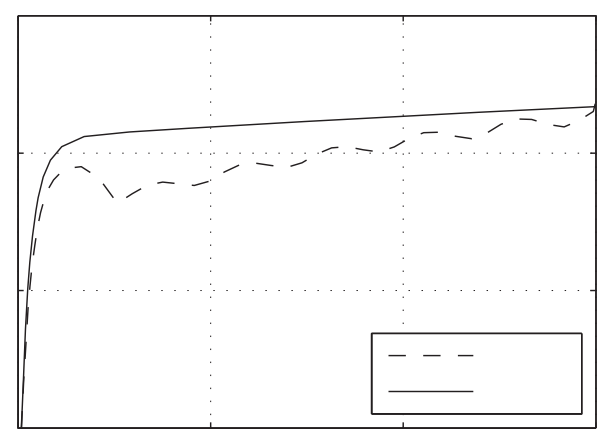

Figure 5: Confined compression test: absolute error of $T_{11}$ during first loading; explicit (ERK2) and semi-implicit (SIRK2) method.

\subsection{Drained triaxial test}

Next, we investigate a drained triaxial test sketched in Figure 7. The initial stresses are $T_{11}=T_{22}=T_{33}=-100 \mathrm{kN} / \mathrm{m}^{2}$, the initial void ratio is $e=0.735$ 


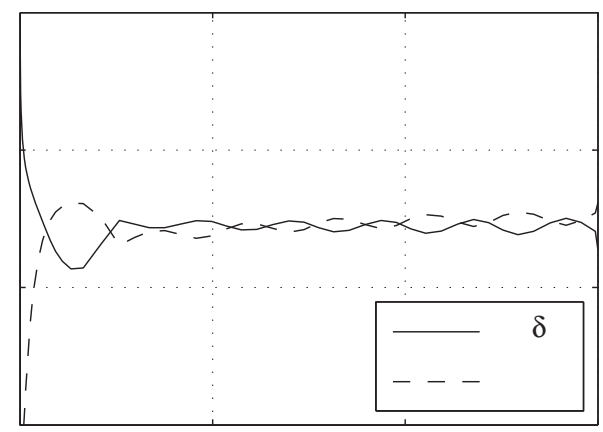

$\varepsilon$

Figure 6: Confined compression test: relative error of $\mathrm{T}_{11}$ and $\delta_{11}$ for ERK2.

and the initial intergranular strains are zero.

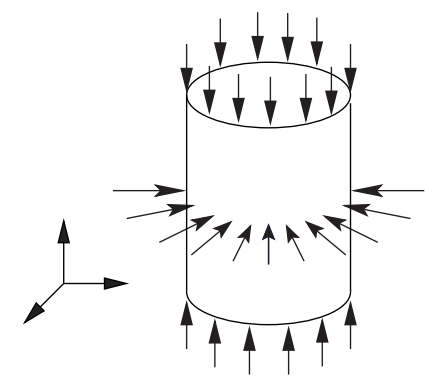

Figure 7: Triaxial test.

The first strain controlled loading was performed in 2 steps, each with $\Delta \varepsilon_{11}=-5 \times 10^{-3}$ and then 4 steps, each with $\Delta \varepsilon_{11}=-1 \times 10^{-2}$, i.e. a total vertical compressive strain of $5 \%$ was applied, see Figure 8 . The unknown horizontal strains $\varepsilon_{22}=\varepsilon_{33}$ were calculated with Newton's method at the end of each step to keep the horizontal stresses constant. As we used the consistent tangent [4] we obtained quadratic convergence. The explicit method needs 330 sub steps in total for the whole first loading, whereas the implicit method achieved a similar relative error (Figure 9) with only 67 sub steps.

The volumetric strain $\varepsilon_{v}=\operatorname{tr} \varepsilon$ is plotted in Figure 10. The stretching is assumed to be constant in each load increment, as usually assumed in finite element calculations. This approximation yields a relatively large difference in volumetric strain between the solution with 6 load steps compared to the exact solution with $10^{4}$ load steps. This error is a shortcoming of separating the 


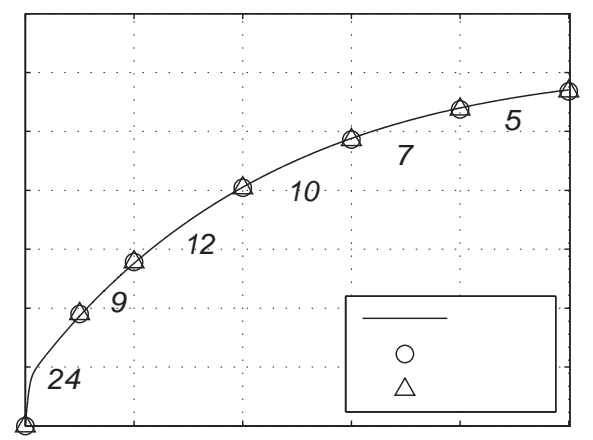

$\varepsilon$

Figure 8: Drained triaxial test: stress-strain relationship; loading steps performed by ERK2 are denoted with circles, loading steps performed by SIRK2 with triangles. The number of sub steps in each load step performed by ERK2 and SIRK2 are printed above (roman characters) and below the curve (italic characters), respectively.

calculation of the equilibrium from the integration of the constitutive relation over certain time windows (operator splitting method) and can only be reduced by increasing the number of load steps.

Figure 11 shows the evolution of the principal values of the intergranular strain of the exact solution during the first loading. Starting from the initial value zero, $\delta$ approaches its extremum, i.e. the sphere with radius $R$, which cannot be exceeded. Further loading deformation is accompanied by a rotation of the stretching tensor, due to the volumetric change of the soil. The evolution equation of the intergranular strain (31) predicts then a rotation of $\boldsymbol{\delta}$, which yields the path on the sphere in Figure 11. Due to this fact, the stiffness of the problem causes a small step size for explicit methods, whereas the semi-implicit method proceeds with quite large sub steps, compare Figures 12 and 13.

After the first loading we perform a stress controlled unloading in two steps. The vertical stress is reduced by $25 \%$ in this unloading. All three unknown strains were calculated with Newton's method. Last, a further strain controlled loading in 2 steps of $\Delta \varepsilon_{11}=-5 \times 10^{-3}$ and 4 steps of $\Delta \varepsilon_{11}=-1 \times 10^{-2}$ followed, see Figure 14. Quadratic convergence of the Newton iterations was achieved in all load steps. The explicit method needs $700 \mathrm{sub}$ steps in total for the whole loading cycle, the semi-implicit one 120 sub steps.

\subsection{Undrained triaxial test}

In an undrained triaxial test the stresses in Figure 7 are total stresses. Considering the pore pressure $u$ as positive at compression the total stress $\mathbf{T}$ and the effective stress $\mathbf{T}^{\prime}$ are related by $T_{i j}=T_{i j}^{\prime}-u \delta_{i j}$. The undrained condition 


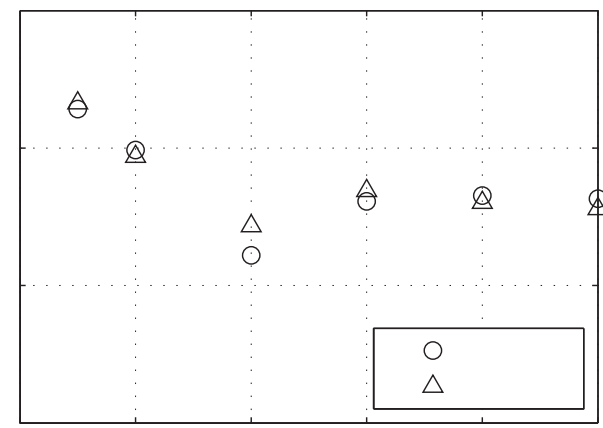

$\varepsilon$

Figure 9: Drained triaxial test: relative error of stress deviator $T_{11}-T_{22}$; circles and triangles denote loading steps with ERK2 and SIRK2, respectively.

$\varepsilon_{v}=0$ yields to $\varepsilon_{22}=\varepsilon_{33}=-\varepsilon_{11} / 2$, cf. e.g. [12]. The integration of the constitutive model with this fully given strain tensor yields the effective stress $\mathbf{T}^{\prime}$. The pore pressure $u$ can be obtained from $\dot{T}_{22}=\dot{T}_{22}^{\prime}+\dot{u}=0$, i.e. $\dot{u}=-\dot{T}_{22}^{\prime}$ with $u(t=0)=u_{0}$.

The initial stresses in our test are $T_{11}=T_{22}=T_{33}=-100 \mathrm{kN} / \mathrm{m}^{2}$, the initial void ratio is $e=0.735$, the initial pore pressure $u_{0}=0$ and the initial intergranular strains are zero.

A loading was performed in one step with $\Delta \varepsilon_{11}=-2 \times 10^{-2}$. The resulting path of the effective stresses is plotted in Figure 15. Again, the explicit method requires much smaller sub steps than the semi-implicit method, especially at the end of the load step, where the soil strength tends to be fully mobilized, compare upper left region in Figure 15. It is obvious that the adaptivity of the integration methods is essential. Otherwise, far too small sub steps have to be chosen for the whole loading step to achieve a required accuracy, especially for the explicit method. In the case of the model with intergranular strain, too large sub steps can even destroy the robustness of the integration, i.e. a too large error in the integration of the intergranular strain will yield to $\delta$-states (far) outside the sphere with radius $R$. Although the vector field of (31) points back to the sphere, numerical solutions do not necessarily return to the sphere.

\subsection{Simple shear test with constant volume}

The deformation occurring in a plane strain simple shear test with constant volume (Figure 16) can be calculated from the shear angle $\gamma_{12}$. The coordinates of the deformed element $\mathbf{x}$ are given by $x_{1}=X_{1}+X_{2} \tan \gamma_{12}, x_{2}=X_{2}$ and $x_{3}=X_{3}$, with the reference coordinates $\mathbf{X}$. 


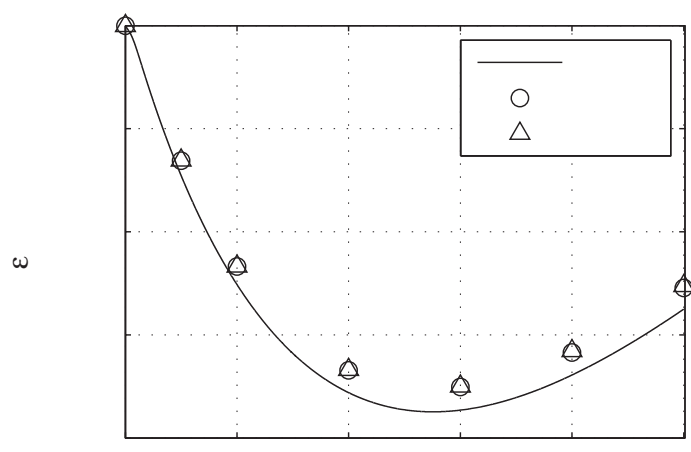

$\varepsilon$

Figure 10: Drained triaxial test: evolution of the volumetric strain; circles and triangles denote loading steps with ERK2 and SIRK2, respectively.

The stretching tensor of this deformation is, compare e.g. [12, Chapt. 3],

$$
\mathbf{D}=\frac{1}{2}\left[\begin{array}{ccc}
0 & \dot{\gamma}_{12} / \cos ^{2} \gamma_{12} & 0 \\
\dot{\gamma}_{12} / \cos ^{2} \gamma_{12} & 0 & 0 \\
0 & 0 & 0
\end{array}\right],
$$

and the spin tensor is

$$
\mathbf{W}=\frac{1}{2}\left[\begin{array}{ccc}
0 & \dot{\gamma}_{12} / \cos ^{2} \gamma_{12} & 0 \\
-\dot{\gamma}_{12} / \cos ^{2} \gamma_{12} & 0 & 0 \\
0 & 0 & 0
\end{array}\right] .
$$

We pass the rotational free strain increment $\Delta \varepsilon=\mathbf{D} \cdot \Delta t$ to the constitutive subroutine and update the stress with (20) and the intergranular strain in the same way.

The initial stresses in our test are $T_{11}=T_{22}=T_{33}=-100 \mathrm{kN} / \mathrm{m}^{2}$, the initial void ratio is $e=0.735$ and the initial intergranular strains are zero. A loading was performed in 5 steps with a total shear angle of $\Delta \gamma_{12}=1 \%$, see Figure 17.

As in all examples above, the explicit method requires more sub steps than the semi-implicit method.

\subsection{Efficiency}

The semi-implicit integration method requires fewer sub steps than the explicit integration method to achieve a similar accuracy in all tests performed above. This is summarized in the plots of Figures 18 and 19. The gap between the total number of sub steps for ERK2 and SIRK2, respectively, increases as the deformation increases. The number of sub steps of the explicit method increases 


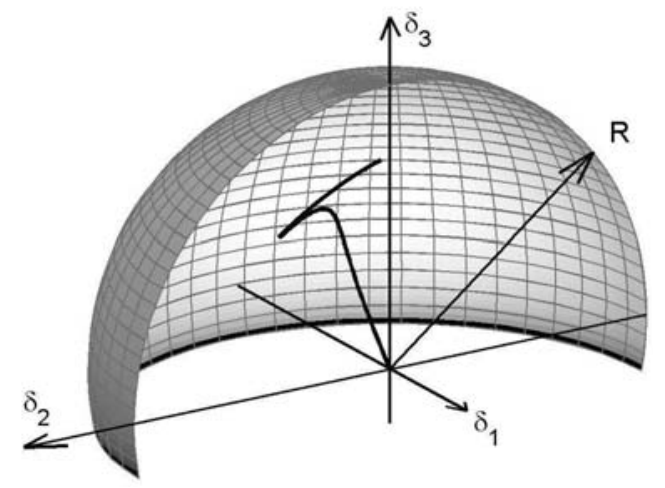

Figure 11: Drained triaxial test: principal values of the intergranular strain during the first loading.

roughly linear with deformation and much more than in the case of the semiimplicit method. However, the computational effort for each of the semi-implicit steps is higher. It is part of the ongoing research of the authors to identify situations, where the semi-implicit method is faster and to find criteria for an automatic switch between the two methods.

\section{Conclusions and Outlook}

An adaptive step size selection is an essential feature of time integration methods. It assures numerical stability and it is the basis for controlling the accuracy of the solution. Our proposed step size control works for explicit and implicit methods. It guarantees numerical stability of the time integration of the investigated constitutive model of the rate type in the framework of a displacement version of the finite element method. Our approach for calculating the consistent tangent numerically, which was proposed in [4] and tested together with an explicit integration method, proves to work for the semi-implicit integration scheme as well. The question whether the semi-implicit or the explicit method is faster cannot be answered a priori. It depends on the situation and has to be investigated in further studies. In particular, we plan to devise an algorithm that automatically switches between the two methods, depending on the stiffness of the problem.

\section{A Hypoplastic model}

For the sake of completeness, we outline the used hypoplastic model and the parameters used for our calculations. Tensors of second order are denoted with bold letters (e.g. $\mathbf{D}, \mathbf{T}, \boldsymbol{\delta}, \mathbf{N}$ ) and tensors of fourth order with calligraphic letters (e.g. $\mathcal{L}, \mathcal{M})$. Different kinds of tensorial multiplication are used: $\mathbf{T D}=$ 


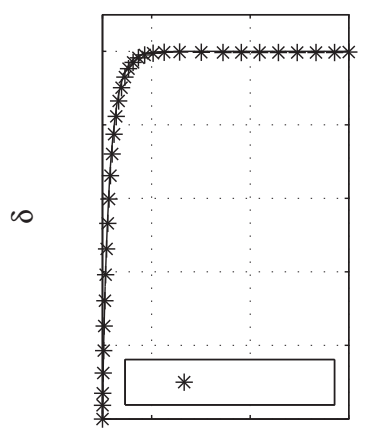

$\varepsilon$

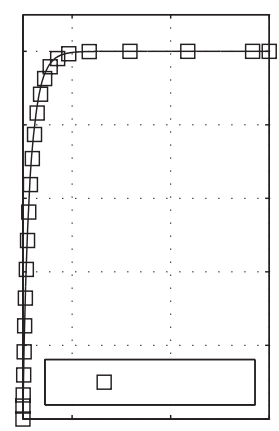

$\varepsilon$

Figure 12: Drained triaxial test: absolute value of the intergranular strain of the first load step from 0 to $-0.5 \%$ during the first loading: Crosses and squares mark sub steps with ERK2 and SIRK2, respectively.

$T_{i j} D_{k l}, \mathbf{T}: \mathbf{D}=T_{i j} D_{i j}, \mathcal{L}: \mathbf{D}=L_{i j k l} D_{k l}, \mathbf{T} \cdot \mathbf{D}=T_{i j} D_{j k}$. The Euclidian norm of a tensor is $\|\mathbf{D}\|=\sqrt{D_{i j} D_{i j}}$. Unit tensors of second and fourth order are denoted by $\mathbf{I}$ and $\mathcal{I}$, respectively.

\section{A.1 Basic model}

The basic hypoplastic model was proposed in [24]

$$
\stackrel{\circ}{\mathbf{T}}=\mathcal{L}(\mathbf{T}, e): \mathbf{D}+\mathbf{N}(\mathbf{T}, e)\|\mathbf{D}\|,
$$

with the linear term

$$
\mathcal{L}=f_{s} \frac{1}{\hat{\mathbf{T}}: \hat{\mathbf{T}}}\left(F^{2} \mathcal{I}+a^{2} \hat{\mathbf{T}} \hat{\mathbf{T}}\right)
$$

and the nonlinear term

$$
\mathbf{N}=f_{s} f_{d} \frac{a F}{\hat{\mathbf{T}}: \hat{\mathbf{T}}}\left(\hat{\mathbf{T}}+\hat{\mathbf{T}}^{*}\right)
$$

The used stress variables are defined as follows

$$
\hat{\mathbf{T}}:=\frac{\mathbf{T}}{\operatorname{tr} \mathbf{T}}, \quad \hat{\mathbf{T}}^{*}:=\hat{\mathbf{T}}-\frac{1}{3} \mathbf{I} .
$$

The factors for pressure and density dependency (barotropy and pyknotropy) 

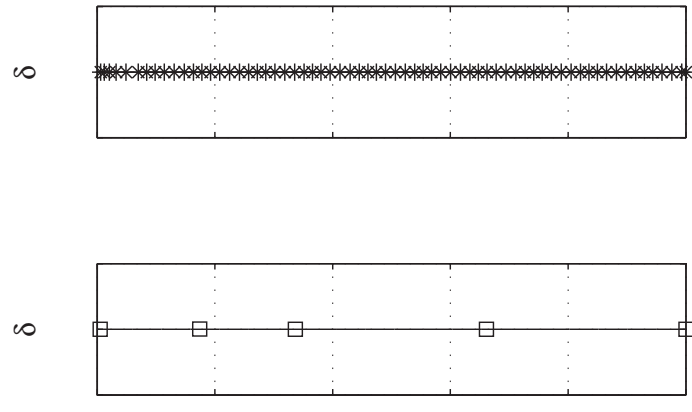

$\varepsilon$

Figure 13: Drained triaxial test: absolute value of the intergranular strain of the last load step from -4 to $-5 \%$ during the first loading: Crosses and squares mark sub steps with ERK2 and SIRK2, respectively.

are given by

$$
\begin{aligned}
a:= & \frac{\sqrt{3}\left(3-\sin \varphi_{c}\right)}{2 \sqrt{2} \sin \varphi_{c}}, \quad f_{d}:=\left(\frac{e-e_{d}}{e_{c}-e_{d}}\right)^{\alpha}, \\
f_{s}:= & \frac{h_{s}}{n}\left(\frac{e_{i}}{e}\right)^{\beta} \frac{1+e_{i}}{e_{i}}\left(\frac{-\operatorname{tr} \mathbf{T}}{h_{s}}\right)^{1-n} \times \\
& {\left[3+a^{2}-a \sqrt{3}\left(\frac{e_{i 0}-e_{d 0}}{e_{c 0}-e_{d 0}}\right)^{\alpha}\right]^{-1} . }
\end{aligned}
$$

The factor $F$ for adapting the deviatoric yield surface to that of MatsuokaNakai is

$$
F:=\sqrt{\frac{1}{8} \tan ^{2} \psi+\frac{2-\tan ^{2} \psi}{2+\sqrt{2} \tan \psi \cos 3 \theta}}-\frac{1}{2 \sqrt{2}} \tan \psi
$$

with

$$
\tan \psi:=\sqrt{3}\left\|\hat{\mathbf{T}}^{*}\right\| \quad \text { and } \quad \cos 3 \theta:=-\sqrt{6} \frac{\operatorname{tr}\left(\hat{\mathbf{T}}^{*} \cdot \hat{\mathbf{T}}^{*} \cdot \hat{\mathbf{T}}^{*}\right)}{\left[\hat{\mathbf{T}}^{*}: \hat{\mathbf{T}}^{*}\right]^{3 / 2}} .
$$

The void ratios are assumed to fulfill the compression law

$$
\frac{e_{i}}{e_{i 0}}=\frac{e_{c}}{e_{c 0}}=\frac{e_{d}}{e_{d 0}}=\exp \left[-\left(\frac{-\operatorname{tr} \mathbf{T}}{h_{s}}\right)^{n}\right] .
$$

This hypoplastic relation has 8 parameters: the critical friction angle $\varphi_{c}$, the granular hardness $h_{s}$, the void ratios $e_{i 0}, e_{c 0}$ and $e_{d 0}$, and the exponents $n, \alpha$ and $\beta$. They can be determined easily from simple index and element tests [8].

Since the mass is assumed to remain constant, the evolution of the void ratio $e$ is described by

$$
\dot{e}=(1+e) \operatorname{tr} \mathbf{D} .
$$




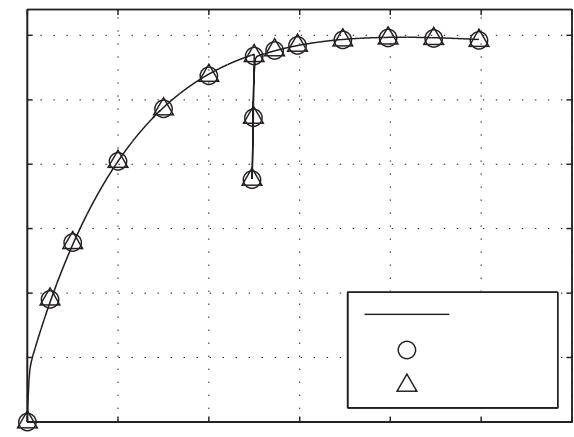

$\varepsilon$

Figure 14: Drained triaxial test: stress-strain relationship in a loadingunloading-reloading cycle: circles and triangles denote loading and unloading steps with ERK2 and SIRK2, respectively.

\section{A.2 Extended hypoplastic model}

The here used extended version of hypoplasticity with intergranular strain was proposed in [20]

The general stress-strain relation is written as

$$
\stackrel{\circ}{\mathbf{T}}=\mathcal{M}: \mathbf{D},
$$

where $\mathcal{M}$ is a fourth order tensor, which represents stiffness and is calculated from the hypoplastic tensors $\mathcal{L}(\mathbf{T}, e)$ and $\mathbf{N}(\mathbf{T}, e)$. The stiffness $\mathcal{M}$ is defined by the following interpolation

$$
\begin{aligned}
\mathcal{M}= & {\left[\rho^{\chi} m_{T}+\left(1-\rho^{\chi}\right) m_{R}\right] \mathcal{L}+} \\
& \begin{cases}\rho^{\chi}\left(1-m_{T}\right) \mathcal{L}: \hat{\boldsymbol{\delta}} \hat{\boldsymbol{\delta}}+\rho^{\chi} \mathbf{N} \hat{\boldsymbol{\delta}} & \text { for } \hat{\boldsymbol{\delta}}: \mathbf{D}>0, \\
\rho^{\chi}\left(m_{R}-m_{T}\right) \mathcal{L}: \hat{\boldsymbol{\delta}} \hat{\boldsymbol{\delta}} & \text { for } \hat{\boldsymbol{\delta}}: \mathbf{D} \leq 0,\end{cases}
\end{aligned}
$$

wherein the normalized magnitude of the intergranular strain $\delta$ is

$$
\rho:=\frac{\|\boldsymbol{\delta}\|}{R} .
$$

Here, $m_{R}, m_{T}, \chi$ and $R$ denote material parameters.

The direction of the intergranular strain $\delta$ is defined as

$$
\hat{\mathcal{\delta}}:=\left\{\begin{array}{ll}
\boldsymbol{\delta} /\|\boldsymbol{\delta}\| & \text { for } \boldsymbol{\delta} \neq \mathbf{0} \\
\mathbf{0} & \text { for } \boldsymbol{\delta}=\mathbf{0}
\end{array} .\right.
$$

The evolution equation of the intergranular strain tensor $\delta$ is postulated

$$
\stackrel{\delta}{\delta}= \begin{cases}\left(\mathcal{I}-\hat{\delta} \hat{\delta} \rho^{\beta_{r}}\right): \mathbf{D} & \text { for } \hat{\boldsymbol{\delta}}: \mathbf{D}>0 \\ \mathbf{D} & \text { for } \hat{\boldsymbol{\delta}}: \mathbf{D} \leq 0\end{cases}
$$




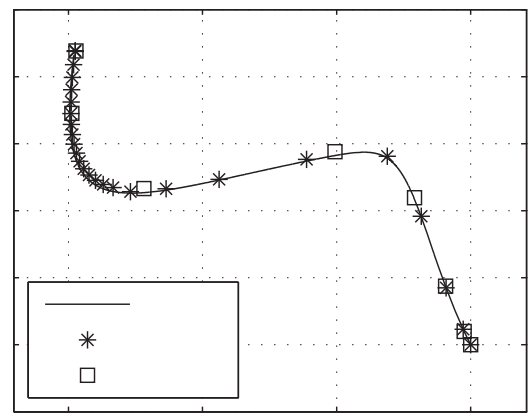

Figure 15: Undrained triaxial test: effective stress path; crosses and squares mark every fifth ERK2 sub step and SIRK2 sub step, respectively.

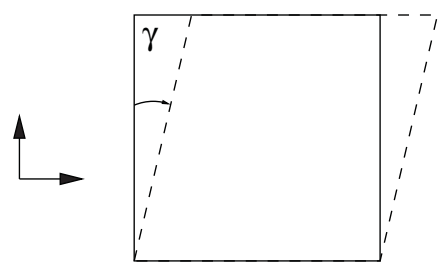

Figure 16: Simple shear test.

where $\delta$ is the objective rate of intergranular strain and the exponent $\beta_{r}$ is a material parameter.

For monotonic continuation of straining with $\mathbf{D} \sim \hat{\mathfrak{\delta}}$, the stiffness is

$$
\mathcal{M}=\mathcal{L}+\mathbf{N} \hat{\delta} .
$$

Note that $\mathbf{D}=\hat{\boldsymbol{\delta}}\|\mathbf{D}\|$ and $\mathbf{N} \hat{\boldsymbol{\delta}}: \mathbf{D}=\mathbf{N}\|\mathbf{D}\|$ in this case. Thus we obtain the basic hypoplastic equation (22).

\section{A.3 Material parameters}

The parameters used in all calculations are listed in Tables 2 and 3.

\begin{tabular}{|c|c|c|c|c|c|c|c|}
\hline$\varphi_{c}\left({ }^{\circ}\right)$ & $h_{s}(\mathrm{kPa})$ & $n$ & $e_{d 0}$ & $e_{c 0}$ & $e_{i 0}$ & $\alpha$ & $\beta$ \\
\hline 33 & $1 \times 10^{6}$ & 0.25 & 0.55 & 0.95 & 1.05 & 0.25 & 1.50 \\
\hline
\end{tabular}

Table 2: Parameters for the basic model 


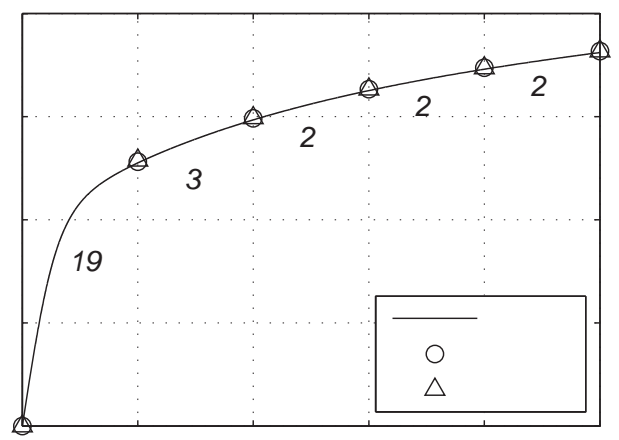

$\gamma$

Figure 17: Simple shear test: loading steps performed by ERK2 are denoted with circles, loading steps performed by SIRK2 with triangles. The number of sub steps in each load step performed by ERK2 and SIRK2 are printed above (roman characters) and below the curve (italic characters), respectively.

\begin{tabular}{|c|c|c|c|c|}
\hline$R$ & $m_{R}$ & $m_{T}$ & $\beta_{r}$ & $\chi$ \\
\hline $1 \times 10^{-4}$ & 5.0 & 2.0 & 0.5 & 6.0 \\
\hline
\end{tabular}

Table 3: Parameters for the extended model

\section{B System of differential equations}

The constitutive subroutine has to integrate the following evolution equations simultaneously in each strain controlled load step for a given strain increment $\Delta \varepsilon$ and a given time increment $\Delta t$, with the initial states $\mathbf{T}(0)$ and $\mathbf{Q}(0)$. Large strain effects are handled by the main program (e.g. the finite element code), thus, the objective rates of the state variables are equal to the time rates in the subroutine. Thus the evolution equations are

$$
\begin{aligned}
\frac{\mathrm{d}}{\mathrm{d} t} \mathbf{T} & =\mathbf{h}(\mathbf{T}, \mathbf{D}, \mathbf{Q}) \\
\frac{\mathrm{d}}{\mathrm{d} t} \mathbf{Q} & =\mathbf{k}(\mathbf{T}, \mathbf{D}, \mathbf{Q}) .
\end{aligned}
$$

The constitutive equation of the stress rate in this paper is given by (27). The rates of the additional state variables $\mathbf{Q}$ are defined in (26) and (31).

The variational equations

$$
\begin{aligned}
\frac{\mathrm{d}}{\mathrm{d} t} \frac{\partial \mathbf{T}}{\partial \mathbf{D}} & =\frac{\partial \mathbf{h}}{\partial \mathbf{T}} \frac{\partial \mathbf{T}}{\partial \mathbf{D}}+\frac{\partial \mathbf{h}}{\partial \mathbf{Q}} \frac{\partial \mathbf{Q}}{\partial \mathbf{D}}+\frac{\partial \mathbf{h}}{\partial \mathbf{D}}, \frac{\partial \mathbf{T}}{\partial \mathbf{D}}(0)=\mathbf{0} \\
\frac{\mathrm{d}}{\mathrm{d} t} \frac{\partial \mathbf{Q}}{\partial \mathbf{D}} & =\frac{\partial \mathbf{k}}{\partial \mathbf{T}} \frac{\partial \mathbf{T}}{\partial \mathbf{D}}+\frac{\partial \mathbf{k}}{\partial \mathbf{Q}} \frac{\partial \mathbf{Q}}{\partial \mathbf{D}}+\frac{\partial \mathbf{k}}{\partial \mathbf{D}}, \frac{\partial \mathbf{Q}}{\partial \mathbf{D}}(0)=\mathbf{0}
\end{aligned}
$$




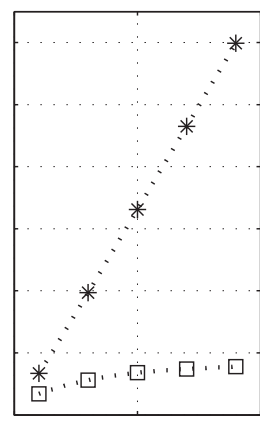

$\varepsilon$

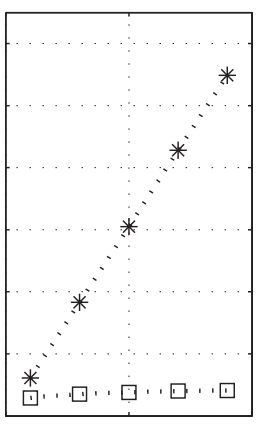

$\varepsilon$

Figure 18: Total number of sub steps against vertical strain $\varepsilon_{11}$ in drained and undrained triaxial test: crosses and squares mark ERK2 and SIRK2, respectively.

are implemented with a numerical approximation [4] to find the Jacobian $\frac{\partial \Delta T_{i j}}{\partial \Delta \varepsilon_{k l}}$. The following abbreviations are used below

$$
B_{i j, k l}=\frac{\partial T_{i j}}{\partial D_{k l}}=\Delta t \cdot \frac{\partial \Delta T_{i j}}{\partial \Delta \varepsilon_{k l}}
$$

and

$$
G_{i, j k}=\frac{\partial Q_{i}}{\partial D_{j k}}
$$

\section{B.1 Assembling of the solution vector}

The variables of the problem are assigned to the components of the solution vector in the following order. The first 6 components of $\mathbf{y}$ are the stress components

$$
\left[y_{1}, y_{2}, \ldots, y_{6}\right]^{\top}=\left[T_{11}, T_{22}, T_{33}, T_{12}, T_{13}, T_{23}\right]^{\top} \text {. }
$$

The next components assemble the additional state variables

$$
\begin{aligned}
{\left[y_{7}, \ldots, y_{13}\right]^{\top} } & =\left[Q_{1}, \ldots, Q_{7}\right]^{\top} \\
& =\left[e, \delta_{11}, \delta_{22}, \delta_{33}, \delta_{12}, \delta_{13}, \delta_{23}\right]^{\top} .
\end{aligned}
$$

The derivatives of the state variables with respect to the stretching

$$
\left[y_{14}, \ldots, y_{49}\right]^{\top}=\left[B_{11,11}, B_{11,22}, \ldots B_{23,23}\right]^{\top}
$$

and

$$
\left[y_{50}, \ldots, y_{91}\right]^{\top}=\left[G_{1,11}, G_{1,22}, \ldots G_{7,23}\right]^{\top}
$$

are collected in the remaining components of the vector $\mathbf{y}$. 


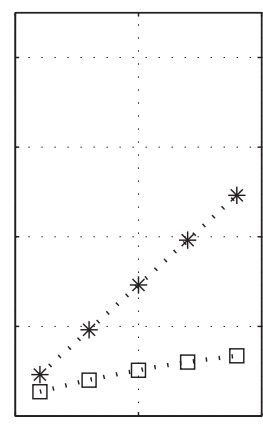

$\varepsilon$

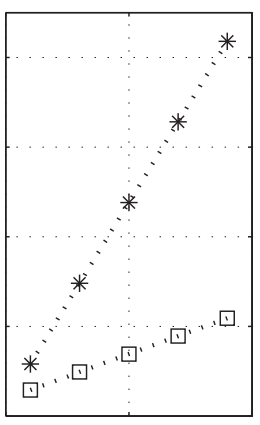

$\gamma$

Figure 19: Total number of sub steps against $\varepsilon_{11}$ in confined compression test and $\gamma_{12}$ in simple shear test: crosses and squares mark ERK2 and SIRK2, respectively.

\section{References}

[1] Batista, D., Royis, P., Doanh, T., 2003. Time-integration of a hypoplastic constitutive equation in finite element modelling. Mathematical and Computer Modelling 37, 615-621.

[2] de Borst, R., Heeres, O., 2002. A unified approach to the implicit integration of standard, non-standard and viscous plasticity models. International Journal for Numerical and Analytical Methods in Geomechanics 26, 1059 1070 .

[3] Desrues, J., Chambon, R., 1993. A new rate type constitutive model for geomaterials. In: Kolymbas, D. (Ed.), Modern Approaches to Plasticity. Elsevier, pp. 309-324.

[4] Fellin, W., Ostermann, A., 2002. Consistent tangent operators for constitutive rate equations. International Journal for Numerical and Analytical Methods in Geomechanics 26, 1213-1233.

[5] Hairer, E., Nørsett, S., Wanner, G., 1993. Solving Ordinary Differential Equations I. Nonstiff Problems, 2nd Edition. Springer, Berlin.

[6] Hairer, E., Wanner, G., 1996. Solving Ordinary Differential Equations II: Stiff and Differential-Algebraic Problems. Springer, Berlin.

[7] Heeres, O., 2001. Modern strategies for the numerical modeling of the cyclic and transient behavior of soils. Ph.D. thesis, TU Delft, DUP Science - Delft University Press. 
[8] Herle, I., 1997. Hypoplastizität und Granulometrie einfacher Korngerüste. Vol. 142 of Veröffentlichung des Institutes für Bodenmechanik und Felsmechanik. Universität Fridericiana in Karlsruhe.

[9] Herle, I., Kolymbas, D., 2004. Hypoplasticity for soils with low friction angles. Computers and Geotechnics 31 (5), 365-373.

[10] Hügel, H., 1995. Prognose von Bodenverformungen. Vol. 136 of Veröffentlichung des Institutes für Bodenmechanik und Felsmechanik. Universität Fridericiana in Karlsruhe.

[11] Kolymbas, D., 1985. A generalized hypoelastic constitutive law. In: Proc. XI Int. Conf. Soil Mechanics and Foundation Engineering, San Francisco. Vol. 5. Balkema, Rotterdam, p. 2626.

[12] Kolymbas, D., 2000. Introduction to Hypoplasticity. No. 1 in Advances in Geotechnical Engineering and Tunnelling. Balkema, Rotterdam.

[13] Kolymbas, D., Herle, I., 2003. Shear and objective stress rates in hypoplasticity. International Journal for Numerical and Analytical Methods in Geomechanics 27, 733-744.

[14] Mašín, D., 2005. A hypoplastic constitutive model for clays. International Journal for Numerical and Analytical Methods in Geomechanics 29 (4), $311-336$.

[15] Mašín, D., 2007. A hypoplastic constitutive model for clays with metastable structure. Canadian Geotechnical Journal 44 (3), 363-375.

[16] Mašín, D., Chambon, R., Desrues, J., 2005. CLoE model modified to predict the behaviour of normally compressed clays. In: Proc. 11th Int. Conference of IACMAG, Turin, Italy. Vol. 2. pp. 417-424.

[17] Mašín, D., Herle, I., 20xx. Improvement of a hypoplastic model to predict clay behaviour under undrained conditions. To appear in Acta Geotechnica.

[18] Mittendorfer, M., 2006. Interne Differentiation nichtlinearer anelastischer Materialmodelle. Master's thesis, University of Innsbruck, Department of Mathematics.

[19] Niemunis, A., 2003. Extended hypoplastic models for soils. Vol. 34 of Schriftreihe des Institutes für Grundbau und Bodenmechanik. RuhrUniversität Bochum.

[20] Niemunis, A., Herle, I., 1997. Hypoplastic model for cohesionless soils with elastic strain range. Mechanics of Cohesive-frictional Materials 2, 279-299.

[21] Roddeman, D., 1997. FEM-implementation of hypoplasticity. Tech. rep., European Union Projekt (ERBCHBGCT 940554), Institut für Geotechnik und Tunnelbau, Universität Innsbruck. 
[22] Tamagnini, C., Viggiani, G., Chambon, R., Desrues, J., 2000. Evaluation of different strategies for the integration of hypoplastic constitutive equations: Application to the CLoE model. Mechanics of Cohesive-frictional Materials $5,263-289$.

[23] Truesdell, C., Noll, W., 1965. The Non-Linear Field Theories of Mechanics. Springer, Berlin, Heidelberg.

[24] von Wolffersdorff, P.-A., 1996. A hypoplastic relation for granular materials with a predefined limit state surface. Mechanics of Cohesive-frictional Materials 1, 251-271.

[25] Weifner, T., Kolymbas, D., 2007. A hypoplastic model for clay and sand. Acta Geotechnica 2 (2), 103-112. 